\title{
NOvA: Current Status and Future Reach
}

\author{
Mathew Muether \\ Fermi National Accelerator Lab, Neutrino Department, P.O. Box 500 MS-220, Batavia, IL 60510 USA
}

\begin{abstract}
$\mathrm{NO} v \mathrm{~A}$, the NuMI Off-Axis $v_{e}$ Appearance experiment will study $v_{\mu} \rightarrow v_{e}$ oscillations, characterized by the mixing angle $\theta_{13}$. A complementary pair of detectors will be constructed $\sim 14$ mrad off beam axis to optimize the energy profile of the neutrinos. This system consists of a surface based $14 \mathrm{kTon}$ liquid scintillatior tracking volume located $810 \mathrm{~km}$ from the main injector source (NuMI) in Ash River, Minnesota and a smaller underground 222 Ton near detector at the Fermi National Accelerator Laboratory (FNAL). The first neutrino signals at the Ash River site are expected soon after the completion of 2012 Fermilab accelerator upgrades. In the meantime, a near detector surface prototype has been completed and neutrinos from two sources at FNAL have been observed using the same highly segmented PVC and liquid scintillator detector system that will be deployed in the full scale experiment. With the recent measurements of $\theta_{13}$ as input, updated sensitivities of $\mathrm{NO} v \mathrm{~A}$ 's capability to ultimately determine the ordering of the neutrino masses and measure $\mathrm{CP}$ violation in neutrino oscillations will be provided. Additionally, design and initial performance characteristics of the surface prototype system along with implications for the full NOvAprogram will be presented.
\end{abstract}

Keywords: neutrino oscillations, neutrino detector technology

PACS: $13.15 .+\mathrm{g}$,

PACS: $14.60 . \mathrm{Pq}$

\section{Introduction}

$\mathrm{NO} v \mathrm{~A}$, the NuMI Off-Axis $v_{e}$ Appearance experiment will study $v_{\mu} \rightarrow v_{e}$ oscillations at a baseline of 810 $\mathrm{km}(\mathrm{L} / \mathrm{E}$ of $400 \mathrm{~km} / \mathrm{GeV})$ beginning in 2013, at which point it will become the flagship experiment for Fermilab. With the recently measured value of $\theta_{13}$ [1], $\mathrm{NO} v \mathrm{~A}$ will have the reach to determine the ordering of the neutrino masses and constrain the $\mathrm{CP}$ violating phase, $\delta$ [2]. Additionally, $\mathrm{NO} v \mathrm{~A}$ will study the differences in oscillations of neutrinos and antineutrinos, as well as make a precision measurement of $\theta_{23}$ by observing the muon neutrino disappearance.

Achieving these goals requires three main components: a far detector with 14 kTons of material capable of suppressing $v_{\mu}$ charge current (CC) and neutral current (NC) backgrounds at the $99 \%$ level and providing good $v_{e}$ detector efficiencies [2], a functionally identical near detector to characterize the beam at the source (rate shown in Figure 1), and an upgrade $700 \mathrm{~kW}$ neutrino beam line. This paper will focus on the experimental design of $\mathrm{NO} v \mathrm{~A}$, present preliminary data from a running prototype, update the project status, and provided current expected physics sensitivities.

\section{Experimental design}

Each component of the $\mathrm{NO} v \mathrm{~A}$ design is described.

\subsection{Beam}

To achieve the goal of the $\mathrm{NO} v \mathrm{~A}$ project, detectors will be placed $14 \mathrm{mrad}$ off-axis to the primary direction of the NuMI (Neutrinos from the Main Injector) source (need reference). As shown in Figure 2, the $v_{\mu}$ flux near the first $v_{\mu} \rightarrow v_{e}$ oscillation maximum at around $2 \mathrm{GeV}$ 


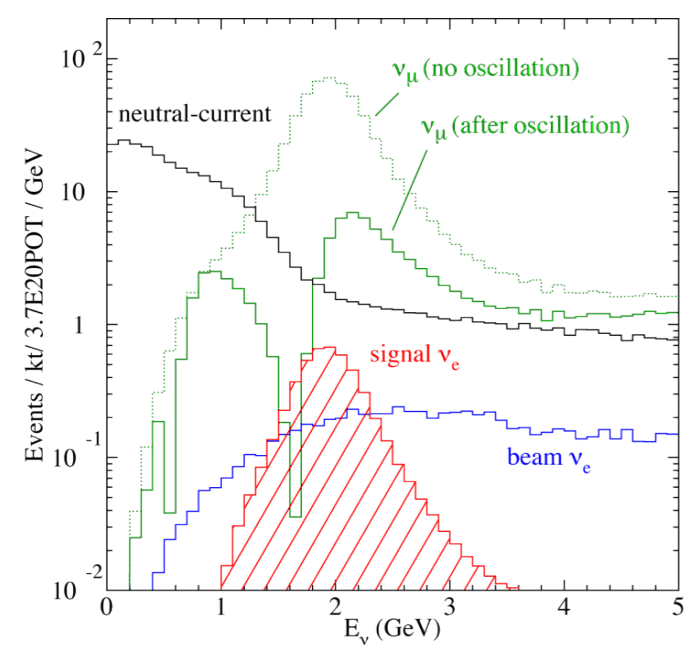

Figure 1: Expected raw signal and background rates for the NOvA far detector. Interaction spectra at $810 \mathrm{~km}, 14 \mathrm{mrad}$ off axis. Oscillations parameters: $\delta \mathrm{m}^{2}=2.5 \times 10^{-3} \mathrm{eV}^{2}, \sin ^{2}\left(2 \theta_{13}\right)=0.01$ [2].

is optimized at this angle [2]. The off-axis beam also reduces high energy neutral current background events. To accommodate the needs of the experiment, the NuMI beam power is being doubled to $700 \mathrm{~kW}$ during the shutdown of the accelerator from March to May 2013.

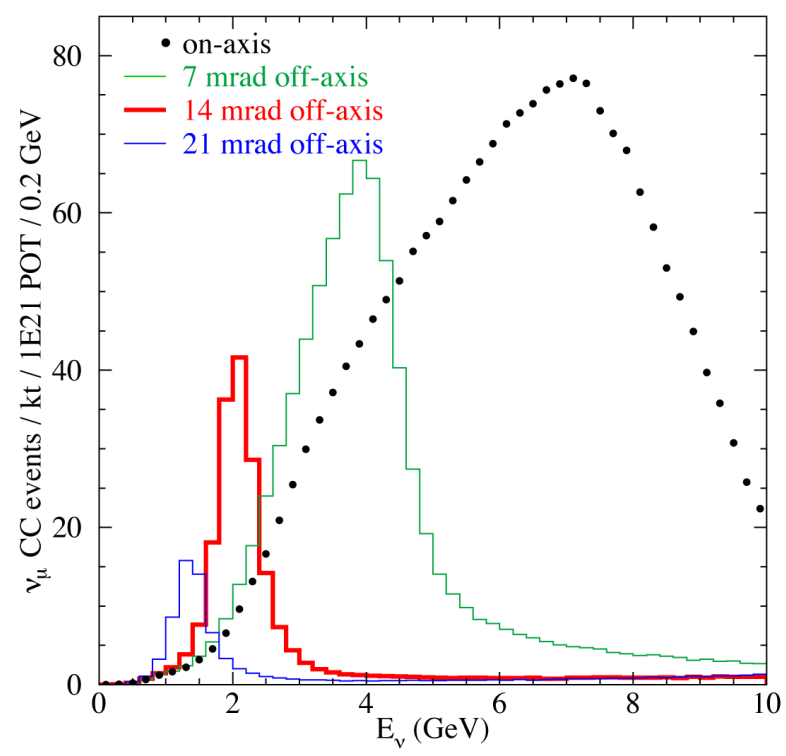

Figure 2: $v_{\mu} \mathrm{CC}$ energy distribution for off-axis angles with medium energy tune [2].

\subsection{Detector Summary}

The NO $v$ A detector system consists of a complementary pair of detectors. Both detectors will be highly segmented tracking calorimeters built entirely from low $\mathrm{Z}$ ( $\sim 0.15$ radiation lengths per layer) $\mathrm{PVC}$, glue, and mineral oil based liquid scintillator with a $65 \%$ active volume [2]. The far detector will be a surface based $14 \mathrm{kTon}$ volume located $810 \mathrm{~km}$ from NuMI in Ash River, Minnesota with about 10 radiation lengths of barite overburden. A smaller 300 Ton unit will be built $1.1 \mathrm{~km}$ from the source at Fermilab in a 105 meters deep underground cavern. Additionally, a 222 ton prototype detector is already in operation on the surface at Fermilab. The full set of detectors is shown in Figure 3.



Figure 3: Image of the NOvAdetectors shown against the the Cathedral of Otranto.

\subsection{Detector modules}

The NOvA detector is built up from modules of extruded $\mathrm{TiO}_{2}$ loaded PVC cells [2]. Each cell is $3.8 \mathrm{~cm}$ by $5.9 \mathrm{~cm}$ in cross section with $90 \%$ reflectivity for light at $430 \mathrm{~nm}$. Extrusions are joined together to produce a sealed module of 32 cells. In the near detector, the modules will be $4.2 \mathrm{~m}$ long while far detector modules are $15.6 \mathrm{~m}$ long. These modules are glued together into alternating planes of horizontal or vertical orientation to create self-supporting 32 layer blocks. $\sim 360,000$ cells make up the $14 \mathrm{kTon}$ far detector.

Internal to each cell is a $0.7 \mathrm{~mm}$ diameter looped fiber routed to an optical connector. The fiber shifts the light collected in the scintillator to $490-550 \mathrm{~nm}$ [2]. The fiber ends are routed through the manifold covered to an optical connector where they are available for single sided readout. $\sim 11,500 \mathrm{~km}$ of fiber is needed for both the far and near detectors.

Once in blocks and positioned, modules are filled with a "home brew" of mineral oil containing 5\% pseudocumene and wavelength shifters to produce $400-450$ 
$\mathrm{nm}$ light. The liquid scintillator makes up $65 \%$ of the total detector mass [2]. The $14 \mathrm{kTon}$ far detector will use over 10.5 million liters of liquid scintillator.

\subsection{Detector Readout}

The light from the fiber ends is incident on Hamamatsu avalanche photodiodes (APD) which have $85 \%$ quantum efficiency for 520-550 nm light [2]. The devices are operated in controlled environmental conditions at $-15^{\circ} \mathrm{C}$ with a gain of 100 . For $\mathrm{NO} v \mathrm{~A}$, a 38 photoelectron (pe) signal from a minimum ionizing particle at the far end of a far detector sized module is expected. 12000 APD arrays are required for the near and far detector design.

The signals from the APDs are processed by frontend electronics (FEBs) which operate in continuous baseline subtraction digitization mode while sampling each channel every $500 \mathrm{~ns}$ [2].

\section{5. $D A Q$}

64 FEBs are fed to a Data Concentrator Module which packages and passes the data in $50 \mu$ s blocks to a processing farm. The data is then buffered at the farm for several seconds at which point a software trigger may be issued to record available data in a specified window. Beam spill and data driven triggers will be available. The entire system is synchronized to GPS and phase locked to its one pulse per second signal. Internal clocking is also available [2].

\subsection{Expected Event}

With this setup, NOvAwill have sensitivities to $v_{\mu}$ charge current quasi-elastic events shown in Figure 4 with a associated Michel electron from the stopping muon and proton recoil inset, $v_{e}$ charge current showers, and neutral current event shown in Figure 4 with a characteristic gap form a photon prior to conversion.

\section{Results from the prototype near detector on the surface (NDOS)}

Six blocks were constructed along with a $1.7 \mathrm{~m}$ muon ranger (Fig. 5) for NDOS. Building the NDOS fully exercised the quality assurance/quality control (QA/QC) techniques in preparation for full production running for the far detector and has been invaluable in understanding production, installation, integration and operations. This detector has been collecting cosmic and neutrino data since October 2010. An additional feature of the NDOS is that it is exposed to both the $0.4 \mathrm{~Hz}$ NuMI (110 mrad off-axis) and 1.2 Hz Booster neutrino (on-axis but

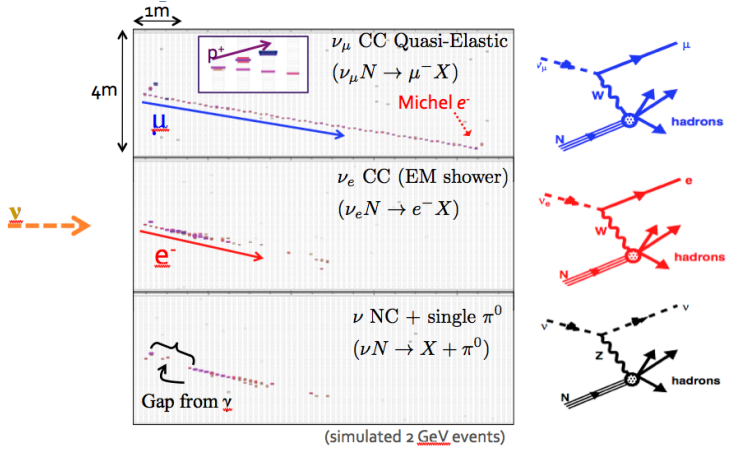

Figure 4: Example of expected NOvAevent topologies.

rotated) beam lines at Fermilab. Prior to the shutdown, 500 microsecond trigger windows were collected from both beams.

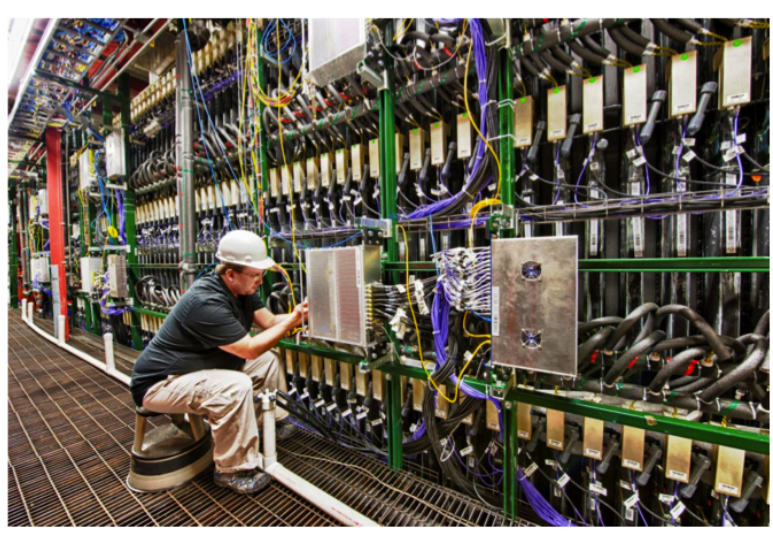

Figure 5: Photograph of the NOvA Near Detector On the Surface.

Analysis of the data from NDOS is in progress. A full suite of available Monte Carlo (masked to behave like our prototype) together with tracking on real data has allowed us to begin to calibrate and reconstruct.

NDOS has collected $5.6 \times 10^{19}$ protons on target (POT) worth of data in reverse horn current beam and $8.4 \times 10^{18}$ POT in forward horn mode from NuMI. Analysis of this sample has yielded 1254 candidate neutrino events with 108 expected cosmic background events. Figure 6 shows an excess of tracks pointing back to the NuMI source over the out-of-time cosmic background and Figure 7 shows this excess as a function of time in the trigger window [4]. Similar distributions have been seen in a Booster neutrino sample of 222 event (with 92 expected background events) from $3 \times 10^{19}$ POT [5].

Additional studies have been performed to understand the energy deposited in the detector and its cell 




Figure 6: Angular track distribution [4].



Figure 7: Timing activity [4].

by cell calibrations. Figure 8 shows the mean ADC value as a function of the distance from the center of the cell from a cosmic muon sample [6]. A sample fit which could be used to calibrate the detector response is shown. Figure 9 shows a sample Michel electron distribution which can be compared against expectation from simulation to provide an electromagnetic energy calibration [3].

\section{Project status}

Module production occurs at an undergraduate driven factory at the University of Minnesota. The factory is

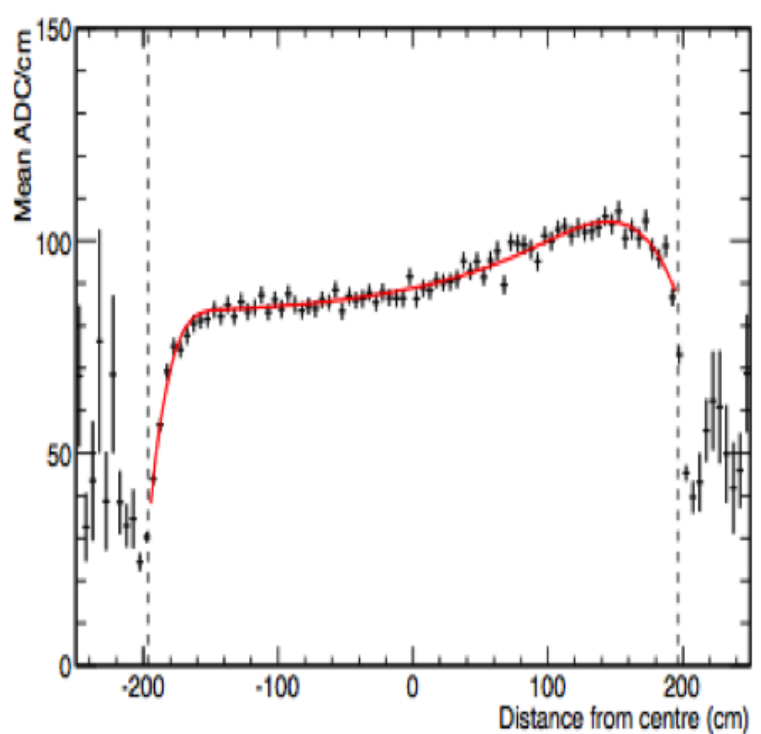

Figure 8: Attenuation from muons [6].

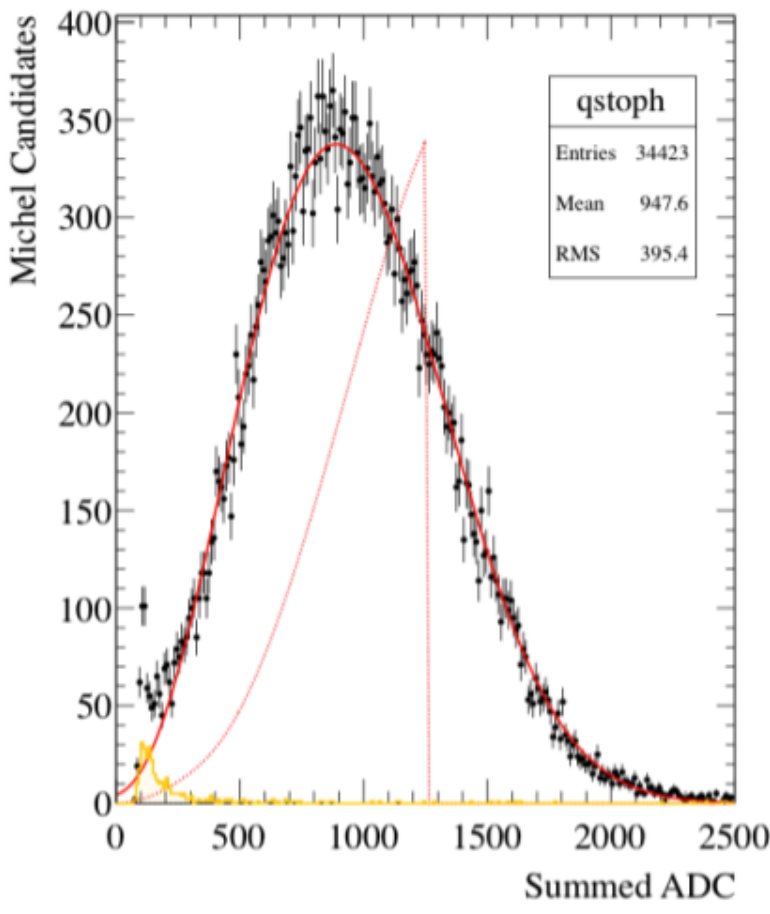

Figure 9: Michel spectrum [3]

reaching full production levels now, necessary to keep pace with the construction of the detector at Ash River.

Beneficial occupancy of the far detector facility was 
obtained in April 2011, and construction of the first blocks is underway. The first block was positioned using the block pivoter on September 10, 2012 and 5 blocks have been placed to date with 1 block being built every 2 weeks. This construction is available via webcam for public viewing [7].

Excavation of the underground cavern for the near detector completed in November 2012 following the beam shutdown and outfitting is on schedule to be ready for near detector blocks in Summer 2013.

The ANU beam upgrades are also progressing. Work is ongoing to turn the Recycler from antiproton to proton ring, shorten Main Injector cycle from 2.2 seconds to 1.33 seconds, and overhaul the NuMI target station for $700 \mathrm{~kW}$ running. This work is scheduled to be complete by spring 2013 .

\section{Physics Sensitivities}

Once complete, the NOvAdetector will be a powerful machine with sensitivity to many neutrino phenomenon as presented in this section. The sensitivities in the section assume $\sin ^{2} 2 \theta_{13}=0.095$, optimization for 4 percent oscillation probability, 10 percent uncertainty on backgrounds, 41 percent $v$ and 48 percent anti- $v$ signal efficiency [8]. Also assumed is the the ramp-up in detector mass and beam power and resulting exposure shown in Figure 10.

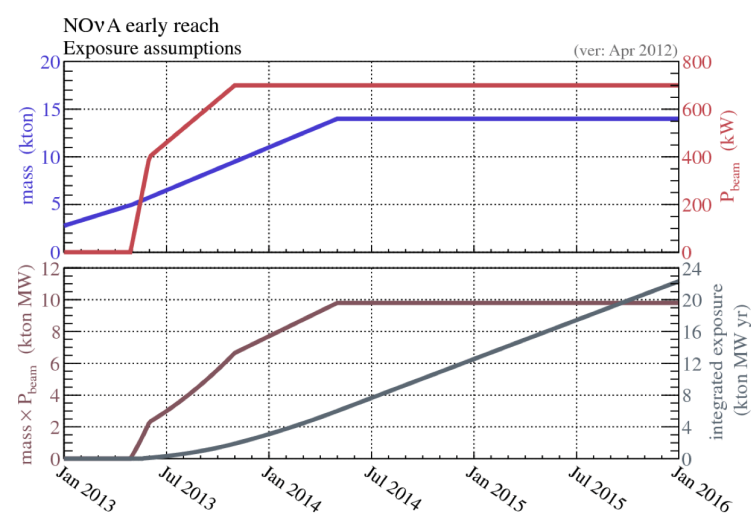

Figure 10: Expected detector mass, NuMI power and exposures [8].

With these assumptions and starting running in $v$ mode, NOvAexpects to achieve an $5 \sigma$ observation of $v_{\mu} \rightarrow v_{e}$ in the first year of running if neutrinos follow the normal hierarchy [8]. This is possible in part to the design of the detector which allows simultaneous running and construction. Anti- $v_{e}$ running could commence at any time to optimize other scenarios of interest. Figure 11 show the details of the $v_{\mu} \rightarrow v_{e}$ sensitivities with respect to time.

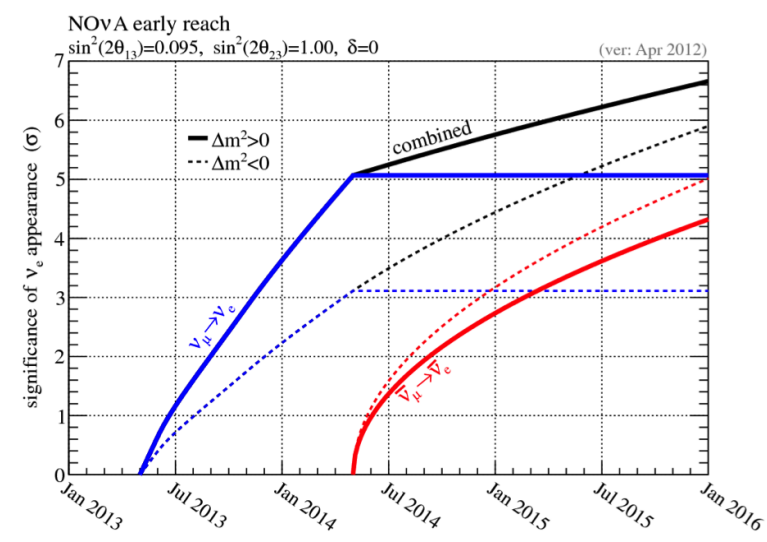

Figure 11: Electron neutrino appearance sensitivities [8].

For additional measurements $\mathrm{NO} v \mathrm{~A}$ will rely on the the comparison of $2 \mathrm{GeV} v_{\mu} \rightarrow v_{e}$ and $\overline{v_{\mu}} \rightarrow \overline{v_{e}}$ probabilities. Figure 12 demonstrates the principle by which NOvA determines the mass hierarchy, measure the $\mathrm{CP}$ phase, and isolate the sign of $\theta_{23}$ [8]. The ellipses show the $\delta \mathrm{cp}$ values and choice of hierarchy that could yield from the oscillation probability measurements given the specified $\sin ^{2}\left(2 \theta_{13}\right)$ value. The blue curves are for the normal hierarchy and the red curves are for the inverted hierarchy. On each ellipse, the choice of the CP phase delta varies as one moves around the ellipse as indicated by the symbols. If $\mathrm{NO} v \mathrm{~A}$ makes a measurement of oscillation probability in each neutrino mode (after 3 years of running in each mode) that yields the starred point with 1- and 2-sigma contours, the hierarchy is resolved, CP phase is constrained for particular values of delta at the 2-sigma level, and $\theta_{23}$ sign is suggested. This assumes maximal mixing in the $\theta_{23}$ octant. This leads to sensitivities for the mass hierarchy determination, CP violation phase, and $\theta_{23}$ octant as shown in Figures $13,14,15$ [8].

\section{Conclusion}

$\mathrm{NO} v \mathrm{~A}$ far detector construction is underway and first data is expected in spring 2013 long with the NuMI $700 \mathrm{~kW}$ upgrades. Near detector excavation is complete and outfitting is scheduled to complete in summer 2013. The NOvA NDOS is taking and analyzing data now. This surface prototype has proved invaluable to 




Figure 12: Biprobability plots described in text [8].

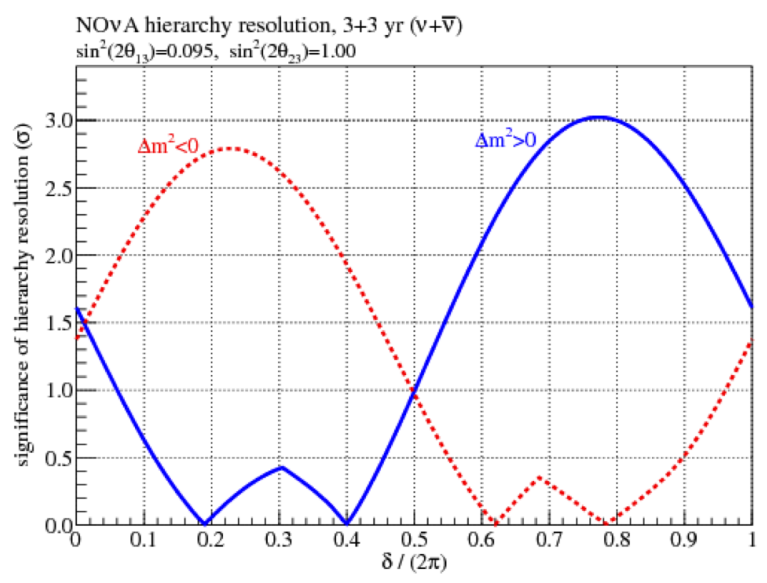

Figure 13: NOvA mass hierarchy sensitivites [8].

all aspects of the experimental program, providing critical feedback for design enhancements and operational experience. The recently measured large value for $\theta_{13}$, is very encouraging for the long term physics reach of $\mathrm{NO} v \mathrm{~A}$ and opens the opportunity to make real contributions in understanding the neutrino especially to mass ordering, $\mathrm{CP}$ violating phase and $\theta_{23}$ octant. The support for $\mathrm{NO} v \mathrm{~A}$ continues to grow with the collaboration now consisting of 150 physicists from 33 institutions in 6 different countries.

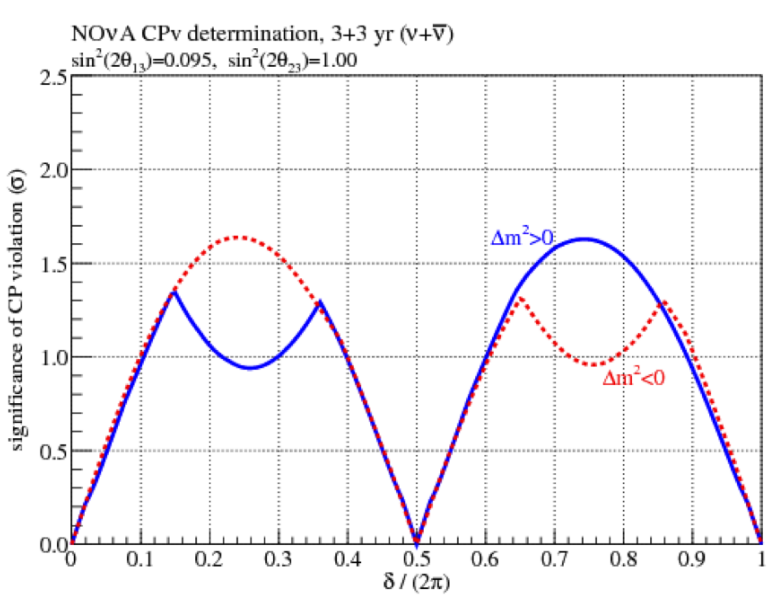

Figure 14: $\mathrm{NO} v \mathrm{~A} \delta \mathrm{CP}$ sensitivites [8].

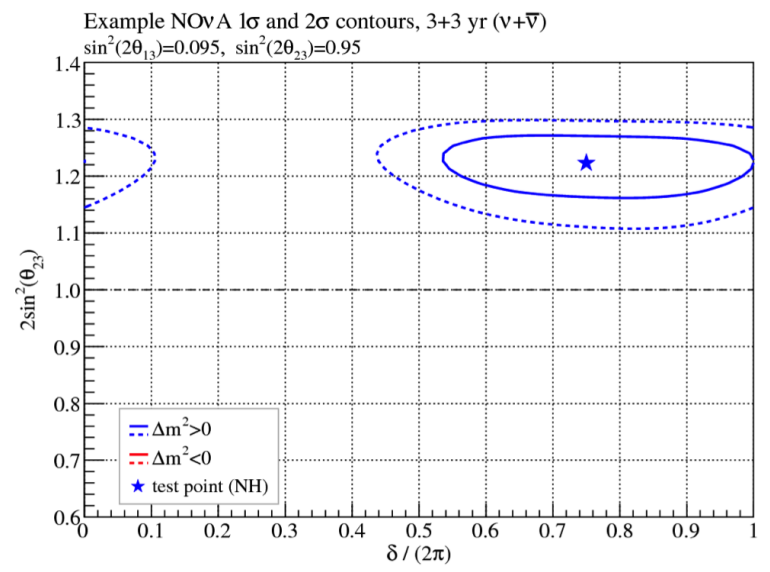

Figure 15: $\mathrm{NO} v \mathrm{~A} \theta_{23}$ sensitivities [8].

\section{References}

[1] Abe K, et al. 2011 Indication of electron neutrino appearance from an accelerator-produced off-axis muon neutrino beam, Preprint arXiv:1106.2822 [hep-ex]

[2] Feldman G, et al. 2007 NOvA Technical Design Report

[3] Messier M 2011 Internal Nova Document NOVA-doc-6023, Low energy event analysis: Michel electrons

[4] Betancourt M 2011 Internal Nova Document NOVA-doc-5874, NuMI Events in NDOS

[5] Johnson C 2011 Internal Nova Document NOVA-doc-5873, Booster Neutrinos in the Nova IPND

[6] Backhouse C 2011 Internal Nova Document NOVA-doc-5931, Cosmic rays for detector calibration

[7] NOvA Far Detector Live Webcam 2012, http://www.fnal.gov/pub/webcams/nova_webcam/

[8] Feldman G, 2012 Internal Nova Document NOVA-doc-7393, NOvA sensitivity plots 